DOI: $10.2478 / \mathrm{v} 10047-011-0030-9$

PHYSICAL AND TECHNICAL ENERGY PROBLEMS

\title{
NOMINAL VOLTAGES OF THE NETWORKS
}

(Part 2)

J. Survilo

Riga Technical University, 1 Kronvalda Blvd., LV-1010, Riga, LATVIA

While in the first stage of electricity supply low voltage is used, the second and third stages exploit higher voltages; therefore, it is of importance in these cases to find the least per unit costs. The next task is to determine the scale of nominal voltages. To solve these tasks, the fed zone should be optimized by size and stage voltage. The author derived an expression which contains zone size and stage voltage dependent and independent terms as well as terms increasing and decreasing with zone size increasing. At the optimum zone size and stage voltage the influence of these terms is balanced. Under certain circumstances it is expedient to install stepdown transformers directly at the customer.

Key words: costs of electricity delivery, electricity supply network, nominal voltage, fed zone.

\section{INTRODUCTION}

In work [1] the discreteness factor of strip $(S)$, bar $(B)$, cruciform square $(S+)$ and $\mathrm{x}$-square $(\mathrm{Sx})$ shaped fed zones were considered, with the costs determined for the $S+$ zone. It was stated that to find the optimum size of a fed zone it is insufficient to consider only the cost of a network itself - it can be found by adding the cost of transformation facility $C_{T f}$ :

$$
C_{z}=C_{n t}+C_{T f} \text {. }
$$

By the transformation facility in the low-voltage case a transformer unit is meant, and in the high-voltage case it is a substation. To compare the fed zone versions of different size, the per unit energy (power) costs are to be used. Further on, per unit power costs will be marked ${ }^{*}$. Thus, for any $\operatorname{cost} C$ we will have:

$$
C^{*}=C / S \text {. }
$$

where $S$ is the power flow through the fed zone transformer facility (a transformer unit or a substation).

\section{DETAILED CONSIDERATION OF NETWORK COSTS}

The calculations show that at a small load density of the fed zone the phase conductor cross-section is so small that it is impossible to obtain the necessary nominal cross-section of the wire, so in these cases we use a given (available) cross-section. As a result, characteristic quantities of a network could change, and the costs per unit derived in [1] become inadequate. At the same time, the fitted 
costs (the first term in expression (38) of [1]) do not change, i.e. they remain considerable both for branch and trunk lines. As concerns the costs dependent on the wire cross-section and electricity losses (the second and third terms in the mentioned expression from [1]), they should be considered separately, since the calculated cross-section of branch lines and trunk lines (or only of the former) might turn out to be too small in view of possible purchases.

To determine whether the cross-section of a phase conductor is in the range of available wires, it is necessary to calculate the required cross-section using an assumed current density $j$. Below, for all the fed zone versions the calculations are performed with proper values of branch line and trunk line cross-sections, $\left(F_{b}\right)$ and $\left(F_{t}\right)$, which are at the beginning of the longest branch line and at the beginning of the trunk line. The initial formula for a cross-section is:

$$
F=I / j .
$$

For the strip fed zone (Fig. 1) we have:

$$
I=\sigma \Delta x \Delta y n=\frac{2 \sigma \Delta x y_{m} n}{2 n+1} ; \quad F=\frac{2 \sigma \Delta x y_{m} n}{(2 n+1) j},
$$

(for $n$ determination in the strip case see [1]). For other fed zone shapes:

$$
n=\frac{x_{m}-\Delta x / 2}{\Delta x} .
$$

For a bar (branch line):

$$
I=\sigma \Delta x \Delta y n=\frac{4 \sigma x_{m} y_{m} n}{(2 N+1)(2 n+1)} ; \quad F_{b}=\frac{4 \sigma x_{m} y_{m} n}{(2 N+1)(2 n+1) j_{b}},
$$

and the same for a trunk line:

$$
I=2 \sigma y_{m} x_{m} ; \quad F_{t}=\frac{2 \sigma y_{m} x_{m}}{j_{t}} .
$$

For square fed zone $S+$ and $S x$ (branch and trunk lines) we will have:

$$
\begin{aligned}
& I=\sigma \Delta x \Delta x n=\sigma \Delta x^{2} n=\frac{4 \sigma x_{m}^{2} n}{(2 n+1)^{2}} ; \quad F_{b}=\frac{4 \sigma x_{m}^{2} n}{(2 n+1)^{2} j_{b}}, \\
& I=\sigma x_{m}^{2} ; \quad F_{t}=\frac{\sigma x_{m}^{2}}{j_{t}} .
\end{aligned}
$$

The original expression for the network costs consists of the five terms:

$$
C_{n t}=C_{a}\left(l_{t}+l_{b}\right)+C_{b}(l F)_{b}+C_{w} \Delta P_{b}+C_{b}(l F)_{t}+C_{w} \Delta P_{t} .
$$
(Fig. 1)

The consideration of $l, l F, \Delta P$ is illustrated by the example for a strip zone 


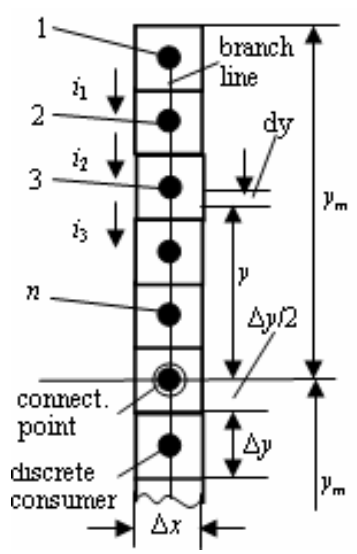

Fig. 1. A strip-shaped fed zone (half area).

We will use the following designations: ()$_{c}$ - continuous, ()$_{d}$ - discrete; discreteness factor $k_{d}=()_{d}:()_{c}$. So we have:

$$
\begin{aligned}
& \left(l F_{g}\right)_{c}=2 \int_{0}^{y_{m}} F_{g} d y=2 F_{g} y_{m} \\
& \left(l F_{g}\right)_{d}=2 F_{g} \Delta y n=2 F_{g} \frac{2 y_{m} n}{2 n+1}=2 F_{g} y_{m} \frac{2 n}{2 n+1} ; \quad k_{d}=\frac{2 n}{2 n+1} .
\end{aligned}
$$

For two wings of a strip and three phases the continuous calculation gives:

$$
\begin{aligned}
& d \Delta P_{F g c}=2 \cdot 3 i^{2}(y) d R_{\Omega}=2 \cdot 3 \sigma^{2} \Delta x^{2}\left(y_{m}-y\right)^{2} \frac{d y}{\gamma F_{g}} ; \\
& \Delta P_{F g c}=\frac{2 \sigma^{2} \Delta x^{2} y_{m}^{3}}{\gamma F_{g}} .
\end{aligned}
$$

The same for discrete calculation:

$$
\begin{aligned}
& \Delta P_{1}=2 \cdot 3 R_{0} \Delta y i_{1}^{2}=\frac{2 \cdot 3 \sigma^{2} \Delta x^{2} \Delta y^{3} 1^{2}}{\gamma F_{g}} ; \quad \Delta P_{2}=\frac{2 \cdot 3 \sigma^{2} \Delta x^{2} \Delta y^{3} 2^{2}}{\gamma F_{g}} ; \ldots \\
& \Delta P_{d}=\sum_{i=1}^{n} \Delta P_{i}=\frac{2 \sigma^{2} \Delta x^{2} y_{m}}{\gamma F_{g}} \frac{4 n(n+1)}{(2 n+1)^{2}} ; \quad k_{d}=\frac{4 n(n+1)}{(2 n+1)^{2}} ; \\
& d U_{\Delta c}=i(y) d R_{\Omega}=\sigma \Delta x\left(y_{m} y\right) \frac{d y}{\gamma F_{g}} ; \quad U_{\Delta F g c}=\frac{\sigma \Delta x y_{m}{ }^{2}}{2 \gamma F_{g}} ; \\
& U_{\Delta 1}=R_{0} i_{1}=\frac{\Delta y}{\gamma F_{g}} \sigma \Delta x 1 \Delta y ; \quad \Delta U_{2}=\frac{\Delta y}{\gamma F_{g}} \sigma \Delta x 2 \Delta y ; \ldots \\
& U_{\Delta F g d}=\sum_{i=1}^{n} \frac{\Delta y}{\gamma F_{g}} \sigma \Delta x \Delta y i=\frac{2 \sigma \Delta x y_{m}^{2}}{\gamma F_{g}} \frac{n(n+1)}{(2 n+1)^{2}} ; \quad k_{d}=\frac{4 n(n+1)}{(2 n+1)^{2}} .
\end{aligned}
$$


Characteristic quantities for the considered fed zones with a given crosssection of phase conductors are shown in Tables 1 and 2 .

Table 1

Characteristic quantities of a strip and a bar with given cross-section $\boldsymbol{F}_{g}$

\begin{tabular}{|c|c|c|c|c|c|}
\hline Case & $\begin{array}{c}l \\
k_{d}\end{array}$ & $\begin{array}{c}(l F) \\
k_{d}\end{array}$ & $\begin{array}{c}\Delta P \\
k_{d}\end{array}$ & $\begin{array}{c}U_{\Delta} \\
k_{d}\end{array}$ & $\begin{array}{l}U_{\delta} \\
k_{d}\end{array}$ \\
\hline$S F_{g}$ & $\begin{array}{l}2 y_{m} \\
\frac{2 n}{2 n+1}\end{array}$ & $\begin{array}{c}2 y_{m} F_{g} \\
\frac{2 n}{2 n+1}\end{array}$ & $\begin{array}{l}\frac{2 \sigma^{2} \Delta x^{2} y_{m}^{3}}{\gamma F_{g}} \\
\frac{4 n(n+1)}{(2 n+1)^{2}}\end{array}$ & $\begin{array}{c}\frac{\sigma \Delta x y_{m}^{2}}{2 \gamma F_{g}} \\
\frac{4 n(n+1)}{(2 n+1)^{2}}\end{array}$ & $\begin{array}{l}\frac{X_{0} \sigma \Delta x y_{m}^{2}}{2} \\
\frac{4 n(n+1)}{(2 n+1)^{2}}\end{array}$ \\
\hline$B b F_{g}$ & $\begin{array}{c}y_{m}(4 N+2) \\
\frac{2 n}{2 n+1}\end{array}$ & $\begin{array}{c}y_{m} F_{g b}(4 N+2) \\
\frac{2 n}{2 n+1}\end{array}$ & $\begin{array}{c}\frac{8 \sigma^{2} x_{m}^{2} y_{m}^{3}}{\gamma_{b} F_{g b}(2 N+1)} \\
\frac{4 n(n+1)}{(2 n+1)^{2}}\end{array}$ & $\begin{array}{c}\frac{\sigma x_{m} y_{m}^{2}}{\gamma_{b} F_{g b}(2 N+1)} \\
\frac{4 n(n+1)}{(2 n+1)^{2}}\end{array}$ & $\begin{array}{l}\frac{X_{0} \sigma x_{m} y_{m}^{2}}{2 n+1} \\
\frac{4 n(n+1)}{(2 n+1)^{2}}\end{array}$ \\
\hline$B t F_{g}$ & $\begin{array}{c}2 x_{m} \\
2 N \\
2 N+1\end{array}$ & $\begin{array}{l}2 x_{m} F_{g t} \\
\frac{2 N}{2 N+1}\end{array}$ & $\begin{array}{l}\frac{4 \sigma^{2} y_{m}^{2} x_{m}^{3}}{\gamma_{t} F_{g t}} \\
\frac{4 N(N+1)}{(2 N+1)^{2}}\end{array}$ & $\begin{array}{c}\frac{\sigma y_{m} x_{m}^{2}}{\gamma_{t} F_{g t}} \\
\frac{4 N(N+1)}{(2 N+1)^{2}}\end{array}$ & $\begin{array}{l}X_{0} \sigma y_{m} x_{m}^{2} \\
\frac{4 N(N+1)}{(2 N+1)^{2}}\end{array}$ \\
\hline
\end{tabular}

Characteristic quantities of square+ and square $\mathbf{x}$ with given cross-section $\boldsymbol{F}_{\boldsymbol{g}}$

Table 2

\begin{tabular}{|c|c|c|c|c|c|}
\hline Case & $\begin{array}{c}l \\
k_{d}\end{array}$ & $\begin{array}{c}(l F) \\
k_{d}\end{array}$ & $\begin{array}{c}\Delta P \\
k_{d}\end{array}$ & $\begin{array}{c}\mathrm{U}_{\Delta} \\
k_{d}\end{array}$ & $\begin{array}{l}U_{\delta} \\
k_{d}\end{array}$ \\
\hline$S+b F_{g}$ & $\begin{array}{l}4 x_{m} n \\
\frac{2 n}{2 n+1}\end{array}$ & $\begin{array}{c}4 x_{m} F_{g b} n \\
\frac{2 n}{2 n+1}\end{array}$ & $\begin{array}{c}\frac{64 \sigma^{2} x_{m}{ }^{5}}{\gamma_{b} F_{g b}} \frac{n^{2}(n+1)^{2}}{(2 n+1)^{5}} \\
1-\frac{1}{2 n(n+1)}\end{array}$ & $\begin{array}{c}\frac{\sigma x_{m}^{3}}{\gamma_{b} F_{g b}(2 n+1)} \\
\frac{4 n^{2}}{(2 n+1)^{2}}\end{array}$ & $\begin{array}{l}\frac{X_{0} \sigma x_{m}^{3}}{2 n+1} \\
\frac{4 n^{2}}{(2 n+1)^{2}}\end{array}$ \\
\hline$S+t F_{g}$ & $\begin{array}{c}4 x_{m} \\
\frac{2 n}{2 n+1}\end{array}$ & $\begin{array}{l}4 x_{m} F_{g t} \\
\frac{2 n}{2 n+1}\end{array}$ & $\begin{aligned} & \frac{32 \sigma^{2} x_{m}^{5}}{5 \gamma_{t} F_{g t}} \\
\approx & \frac{16 n^{4}+20 n^{3}}{(2 n+1)^{4}}\end{aligned}$ & $\begin{array}{l}\frac{2 \sigma x_{m}^{3}}{3 \gamma_{t} F_{g t}} \\
\frac{4 n(n+1)}{(2 n+1)^{2}}\end{array}$ & $\begin{array}{l}\frac{2 X_{0} \sigma x_{m}^{3}}{3} \\
\frac{4 n(n+1)}{(2 n+1)^{2}}\end{array}$ \\
\hline$S \times b F_{g}$ & $\begin{array}{l}4 x_{m} n \\
\frac{2 n}{2 n+1}\end{array}$ & $\begin{array}{c}4 x_{m} F_{g b} n \\
\frac{2 n}{2 n+1}\end{array}$ & $\begin{array}{c}\approx \frac{64 \sigma^{2} x_{m}^{5}}{\gamma_{b} F_{g b}} \frac{n^{4}+8 n^{3} / 3}{(2 n+1)^{5}} \\
\approx \frac{n^{4}+4 n^{3}}{n^{4}+8 n^{3} / 3}\end{array}$ & $\begin{array}{c}\frac{4 \sigma x_{m}{ }^{3} n(n+0.5)}{\gamma_{b} F_{g b}(2 n+1)^{3}} \\
\frac{n+1}{n+0.5}\end{array}$ & $\begin{array}{l}\frac{X_{0} \sigma x_{m}{ }^{3}}{2 n+1} \\
\frac{4 n(n+1)}{(2 n+1)^{2}}\end{array}$ \\
\hline$S \mathrm{x} t F_{g}$ & $\begin{array}{l}4 \sqrt{2} x_{m} \\
\frac{2 n}{2 n+1}\end{array}$ & $\begin{array}{c}4 \sqrt{2} x_{m} F_{g t} \\
\frac{2 n}{2 n+1}\end{array}$ & $\begin{array}{c}\frac{12 \sqrt{2} \sigma^{2} x_{m}{ }^{5}}{5 \gamma_{t} F_{g t}} \\
\frac{16}{3} \frac{n(n+1)(2 n+1)\left(3 n^{2}+3 n-1\right)}{(2 n+1)^{5}}\end{array}$ & $\begin{array}{l}\frac{\sqrt{2} \sigma x_{m}^{3}}{3 \gamma_{t} F_{g t}} \\
\frac{4 n(n+1)}{(2 n+1)^{2}}\end{array}$ & $\begin{array}{l}\frac{\sqrt{2} X_{0} \sigma x_{m}^{3}}{3} \\
\frac{4 n(n+1)}{(2 n+1)^{2}}\end{array}$ \\
\hline
\end{tabular}


Remembering that

$$
S=\sqrt{3} U I ; \quad I=4 \sigma x_{m}^{2} ; \quad \sigma=\frac{\sigma_{s}}{\sqrt{3} U},
$$

the per unit value of the first term in expression (10) for fed zones $S+$ and $S \mathrm{x}$, based on Table 2 of the present paper and on expressions (34)-(38) of [1], is:

$$
C_{a l}^{*}=\frac{C_{a}\left(l_{b}+l_{t}\right)}{S} \approx \frac{C_{a}}{\sigma_{s} \Delta x} .
$$

The remaining terms of expression (10) are determined depending on the formulas for $(l F)$ and $\Delta P$ in Tables 3, 4 of [1] and Table 2 above. The $2^{\text {nd }}-5^{\text {th }}$ terms of expression (10) will look as

$$
C_{b b-}{ }^{*}=C_{b}(l F)_{b} ; C_{w b-}{ }^{*}=C_{w} \Delta P_{b} ; C_{b t-}{ }^{*}=C_{b}(l F)_{t} ; C_{w t-}{ }^{*}=C_{w} \Delta P_{t} .
$$

The third index indicates how the cross-section is calculated, for example: $C_{w b j}$ are the costs of power losses (w) of a branch line (b) with cross-section calculated for constant current density $(j) ; C_{--F}-$ indicates that the cross-section is constant along the entire line; $C_{--F g}-$ indicates that the cross-section of a line is known (see Table 2).

Per unit costs are determined observing the tables above and data of [1] and expressions (2), (11), (17).

For fed zone $\mathrm{S}+$ we have:

$$
\begin{aligned}
& C_{b b j}^{*}=\frac{C_{b} x_{m}}{3 \sqrt{3} j_{b} U} \frac{4 n(n+1)}{(2 n+1)^{2}} ; \quad C_{w b j}{ }^{*}=\frac{C_{w} j_{b} x_{m}}{\sqrt{3} \gamma_{b} U} \frac{4 n(n+1)}{(2 n+1)^{2}} ; \\
& C_{b b F}{ }^{*}=\frac{2 C_{b} x_{m}}{3 \sqrt{3} j_{b} U}\left(\frac{4 n(n+1)}{(2 n+1)^{2}}-\frac{6 n(n+1)}{(2 n+1)^{3}}\right) ; \\
& C_{w b F}{ }^{*}=\frac{2 C_{w} j_{b} x_{m}}{3 \sqrt{3} \gamma_{b} U}\left(\frac{4 n(n+1)}{(2 n+1)^{2}}-\frac{6 n}{(2 n+1)^{3}}\right) ; \\
& C_{b b F g}{ }^{*}=\frac{C_{b} F_{g b} n}{\sigma_{s} x_{m}} \frac{2 n}{2 n+1} ; \\
& C_{w b F g}{ }^{*}=\frac{16 C_{w} \sigma_{s} x_{m}^{3}}{3 \gamma_{b} F_{g b} U^{2}} \frac{n^{2}(n+1)^{2}}{(2 n+1)^{5}}\left(1-\frac{1}{2 n(n+1)}\right) ; \\
& C_{b t j}{ }^{*}=\frac{2 C_{b} x_{m}}{3 \sqrt{3} j_{t} U} \frac{4 n(n+1)}{(2 n+1)^{2}} ; \\
& C_{w t j}{ }^{*}=\frac{2 C_{w} j_{t} x_{m}}{\sqrt{3} \gamma_{t} U} \frac{4 n(n+1)}{(2 n+1)^{2}} ; \quad C_{b t F}{ }^{*}=\frac{C_{b} x_{m}}{\sqrt{3} j_{t} U} \frac{8 n^{2}(n+1)}{(2 n+1)^{3}} ;
\end{aligned}
$$




$$
\begin{aligned}
& C_{w t F}{ }^{*}=\frac{8 C_{w} j_{t} x_{m}}{5 \sqrt{3} \gamma_{t} U} \frac{15}{2}\left(\frac{1}{3}-\frac{n(n+1)}{(2 n+1)^{2}}+\frac{3 n^{2}+3 n-1}{15(2 n+1)^{2}}\right) ; \\
& C_{b t F g}{ }^{*}=\frac{C_{b} F_{g t}}{\sigma_{s} x_{m}} \frac{2 n}{2 n+1} ; \quad C_{w t F g}^{*}=\frac{8 C_{w} \sigma_{s} x_{m}^{3}}{15 \gamma_{t} F_{g t} U^{2}} \frac{16 n^{4}+20 n^{3}}{(2 n+1)^{4}} .
\end{aligned}
$$

In the $S \mathrm{x}$ fed zone the cost components $C_{b b j}{ }^{*} ; C_{w b j}{ }^{*} ; C_{b b F}{ }^{*} ; C_{w b F}{ }^{*} ; C_{b b F g}{ }^{*}$ remain the same as in the $\mathrm{S}+$ fed zone; the other components in the former are:

$$
\begin{aligned}
& C_{w b F g}{ }^{*}=\frac{16 C_{w} \sigma_{s} x_{m}^{3}}{3 \gamma_{b} F_{g b} U^{2}} \frac{n^{4}+4 n^{3}}{(2 n+1)^{5}} ; \quad C_{b t j}{ }^{*}=\frac{\sqrt{2} C_{b} x_{m}}{3 \sqrt{3} j_{t} U} \frac{4 n(n+1)}{(2 n+1)^{2}} ; \\
& C_{w t j}{ }^{*}=\frac{\sqrt{2} C_{w} j_{t} x_{m}}{\sqrt{3} \gamma_{t} U} \frac{4 n(n+1)}{(2 n+1)^{2}} ; \quad C_{b t F}{ }^{*}=\frac{\sqrt{2} C_{b} x_{m}}{\sqrt{3} j_{t} U} \frac{2 n}{2 n+1} ; \\
& C_{w t F}{ }^{*}=\frac{3 \sqrt{2} C_{w} j_{t} x_{m}}{5 \sqrt{3} \gamma_{t} U} \frac{4(n+1)\left(3 n^{2}+3 n-1\right)}{3 n(2 n+1)^{5}} ; \\
& C_{b t F g}{ }^{*}=\frac{\sqrt{2} C_{b} F_{g t}}{\sigma_{s} x_{m}} \frac{2 n}{2 n+1} ; \\
& C_{w t F g}{ }^{*}=\frac{3 \sqrt{2} C_{w} \sigma_{s} x_{m}^{3}}{15 \gamma_{t} F_{g t} U^{2}} \frac{16}{3} \frac{n(n+1)(2 n+1)\left(3 n^{2}+3 n-1\right)}{(2 n+1)^{5}} .
\end{aligned}
$$

\section{COSTS OF TRANSFORMATION FACILITIES}

When defining the costs of a transformation facility it should be taken into account that all its variable elements depend mainly on the transformer capacity $\left(S_{T}\right)$, which is chosen according to a graded scale. To apply relevant formulas, the scale of transformer capacities should be considered continuous:

$$
S_{T}=S / \beta,
$$

where $\beta$ is the transformer utilization factor.

If two transformers are installed and operating with a proportional load, then:

$$
\begin{aligned}
& S_{T 1}+S_{T 2}=S / \beta ; \quad S_{T 1}=S_{1} / \beta=\kappa_{1} S / \beta ; \\
& S_{T 2}=S_{2} / \beta=\kappa_{2} S / \beta ; \quad \kappa_{1}+\kappa_{2}=1,
\end{aligned}
$$

where $S_{1}$ and $S_{2}$ are the respective proportions of power directed through the first and second transformers,

$\kappa_{1}$ and $\kappa_{2}$ are the respective ratios for power $S_{1}$ and $S_{2}$.

For a transformer facility we assume: 1) the number of transformers can be one or more (e.g. two); 2) one or more transformers can be operating; 3 ) the rated capacity of the substation can be equal to the maximum annual capacity (unless otherwise specified, e.g. in the case when some reserve should be secured). 
The annual costs are assumed to consist of the bank payments $\left(C_{T f b}\right)$, the cost of transformer load losses $\left(\Delta P_{l d}\right)$, and the cost of no-load losses $\left(\Delta P_{n l}\right)$

$$
\begin{aligned}
& C_{T f}=C_{T f b}+C_{T l}+C_{T n l} ; \\
& C_{T f b}=C_{p f} K_{T f} ; \quad C_{T l}=C_{w} \Delta P_{l d} ; \quad C_{T n l}=C_{w n l} \Delta P_{n l},
\end{aligned}
$$

where $K_{T f}$ is the cost of transformer facility, which consists of a constant (independent of the facility capacity) fitted portion $\left(K_{0 T f}\right)$ and of a variable (dependent on the substation capacity) portion $\left(K_{v}\right)$. In turn, $K_{0 T f}$ consists of the constant costs of transformer facility $\left(K_{p r}\right)$ and of constant transformer costs $K_{0 T \Sigma}$; the term $K_{v}$ is proportional (with $k_{c T}$ being the proportionality factor) to the total transformer capacity $S_{T \Sigma}$, which can be determined through the total transformer utilization factor $\beta=S / S_{T \Sigma}$. All this can be described by the following set of formulas:

$$
\begin{aligned}
& K_{T f}=K_{0 T f}+K_{v} ; \quad K_{0 T f}=K_{p r}+K_{0 T \Sigma} ; \quad K_{v}=k_{c T} S_{T \Sigma}=k_{c T} S / \beta ; \\
& C_{T f b}=C_{0 T f}+C_{c T} ; \quad C_{0 T f}=C_{p f} K_{0 T f} ; \quad C_{c T}=C_{p f} K_{v} .
\end{aligned}
$$

The loss factor $C_{w}$ can be found in [1]. The no-load loss factor is:

$$
C_{w n l}=\left(\beta^{\prime} T+\beta^{\prime \prime}\right) T,
$$

where $\beta^{\prime}, \beta^{\prime \prime}$ are the respective costs of line power losses and of the power during the peak consumption hours;

$T \quad$ is the duration of a year.

The load and no-load losses (see [2]) can be defined as

$$
\Delta P_{l d}=k_{l d} S^{\alpha_{l d}} ; \quad \Delta P_{n l}=k_{n l} S^{\alpha_{n l}} .
$$

To determine the $k_{l d}, \alpha_{l d}, k_{n l}, \alpha_{n l}$ coefficients, the source base transformer should have capacity $S_{0}$ with load and no-load losses $\Delta P_{l d 0}$ and $\Delta P_{l n 0}$. Therefore:

$$
k_{l d}=\frac{\Delta P_{l d 0}}{S_{0}^{\alpha_{l d}}} ; \quad k_{n l}=\frac{\Delta P_{n l 0}}{S_{0}^{\alpha_{n l}}} .
$$

Later on, the coefficients should be adjusted so that formulas (26) describe the transformers of the same type but with different rated power.

When a transformer with utilization factor $\beta$ is considered, the load losses can be defined as

$$
\Delta P_{l d \beta}=\beta^{2} \Delta P_{l d}=\beta^{2} k_{l d} S_{T}^{\alpha_{l d}} .
$$

If two transformers with utilization factor $\beta$ are in service, then

$$
\Delta P_{l d \Sigma}=\beta^{2} k_{l d}\left(S_{T 1}^{\alpha_{l d}}+S_{T 2}^{\alpha_{l d}}\right) .
$$

The no-load summary losses in the two-transformer case are:

$$
\Delta P_{n l \Sigma}=k_{n l}\left(S_{T 1}^{\alpha_{n l}}+S_{T 2}^{\alpha_{n l}}\right) .
$$


Now (22) can be rewritten as

$$
\begin{aligned}
C_{T f}= & C_{p f} K_{0 T f}+C_{p f} k_{c T} S / \beta+C_{w} \beta^{2} k_{l d}\left(S_{T 1}^{\alpha_{l d}}+S_{T 2}{ }^{\alpha_{l d}}\right)+ \\
& +C_{w n l} k_{n l}\left(S_{T 1} \alpha_{n l}+S_{T 2}{ }^{\alpha_{n l}}\right) .
\end{aligned}
$$

Per unit terms of (31), observing (15) and (21)-(30), can be concretized as

$$
\begin{aligned}
& C_{0 T f}{ }^{*}=\frac{C_{p f} K_{0 T f}}{4 \sigma_{s} x_{m}^{2}} ; \quad C_{c T}{ }^{*}=\frac{C_{p f} k_{c T}}{\beta} ; \\
& C_{T l}{ }^{*}=\frac{C_{w} k_{l d} \beta^{2-\alpha_{l d}}}{\left(4 \sigma_{s} x_{m}^{2}\right)^{1-\alpha_{l d}}}\left(\kappa_{1}^{\alpha_{l d}}+\kappa_{2}{ }^{\alpha_{l d}}\right) ; \\
& C_{T n l}^{*}=\frac{C_{w n l} k_{n l} \beta^{-\alpha_{n l}}}{\left(4 \sigma_{s} x_{m}^{2}\right)^{1-\alpha_{n l}}}\left(\kappa_{1}^{\alpha_{n l}}+\kappa_{2}^{\alpha_{n l}}\right) .
\end{aligned}
$$

\section{THE LOW VOLTAGE FED ZONE}

Based on expressions (1), (16), (18), (19), and (32), we can define the summary fed zone costs. The formula will look different, depending on how the terms of a network are calculated. Below, the given cross-section $F_{g}$ is used. The summary per unit costs of the low voltage $\mathrm{S}+$ fed zone are:

$$
\begin{aligned}
C_{z}{ }^{*}= & C_{a l}{ }^{*}+C_{b b F g}{ }^{*}+C_{b t F g}{ }^{*}+C_{w b F g}{ }^{*}+ \\
& +C_{w t F g}{ }^{*}+C_{0 T f}{ }^{*}+C_{c T}{ }^{*}+C_{T l}{ }^{*}+C_{T n l}{ }^{*}= \\
& =\frac{C_{a}}{\sigma_{s} \Delta x}+\frac{C_{b} F_{g b} n}{\sigma_{s} x_{m}} \frac{2 n}{2 n+1}+\frac{C_{b} F_{g t}}{\sigma_{s} x_{m}} \frac{2 n}{2 n+1}+ \\
& +\frac{16 C_{w} \sigma_{s} x_{m}{ }^{3}}{3 \gamma_{b} F_{g b} U_{I}{ }^{2}} \frac{n^{2}(n+1)^{2}}{(2 n+1)^{5}} \cdot\left(1-\frac{1}{2 n(n+1)}\right)+ \\
& +\frac{8 C_{w} \sigma_{s} x_{m}{ }^{3}}{15 \gamma_{t} F_{g t} U_{I}{ }^{2}} \frac{16 n^{4}+20 n^{3}}{(2 N+1)^{4}}+ \\
& +\frac{C_{p f} K_{0 T f}\left(U_{I I}\right)}{4 \sigma_{s} x_{m}{ }^{2}}+\frac{C_{p f} k_{c T}\left(U_{I I}\right)}{\beta}+ \\
& +\frac{C_{w} k_{l d} \beta^{2-\alpha_{l d}}}{\left(4 \sigma_{s} x_{m}{ }^{2}\right)^{1-\alpha_{l d}}}\left(\kappa_{1}^{\alpha_{l d}}+\kappa_{2}{ }^{\alpha_{l d}}\right)+ \\
& +\frac{C_{w n l} k_{n l} \beta^{-\alpha_{n l}}}{\left(4 \sigma_{s} x_{m}{ }^{2}\right)^{1-\alpha_{n l}}}\left(\kappa_{1}^{\alpha_{n l}}+\kappa_{2}{ }^{\left.\alpha_{n l}\right),}\right.
\end{aligned}
$$

where $U_{I}$ is the nominal low voltage,

$U_{I I}$ is the nominal voltage of a next (the medium-voltage) stage, e.g. $6 ; 10$; $20 \mathrm{kV}$. 
The transformer facility cost depends mainly on the transformer's higher voltage side. To a lesser degree, the same relates to factors $k_{l d}, k_{n l}, \alpha_{l d}, \alpha_{n l}$ (not considered below).

When option $F$ (see [1]) is used for fed zone $\mathrm{S}+$, the summary cost is:

$$
\begin{aligned}
C_{z}^{*}= & \frac{C_{a}}{\sigma_{s} \Delta x}+\frac{2 C_{b} x_{m}}{3 \sqrt{3} j_{b} U_{I}}\left(\frac{4 n(n+1)}{(2 n+1)^{2}}-\frac{6 n(n+1)}{(2 n+1)^{3}}\right)+\frac{C_{b} x_{m}}{\sqrt{3} j_{t} U_{I}} \frac{8 n^{2}(n+1)}{(2 n+1)^{3}}+ \\
& +\frac{2 C_{w} j_{b} x_{m}}{3 \sqrt{3} \gamma_{b} U_{I}}\left(\frac{4 n(n+1)}{(2 n+1)^{2}}-\frac{6 n}{(2 n+1)^{3}}\right)+ \\
& +\frac{8 C_{w} j_{t} x_{m}}{\sqrt{3} \gamma_{t} U_{I}} \frac{3}{2}\left(\frac{1}{3}-\frac{n(n+1)}{(2 n+1)^{2}}\right)+\frac{3 n^{2}+3 n-1}{15(2 n+1)^{2}} \\
& +\frac{C_{p f} K_{0 T f}\left(U_{I I}\right)}{4 \sigma_{s} x_{m}{ }^{2}}+\frac{C_{p f} k_{c T}\left(U_{I I}\right)}{\beta}+ \\
& +\frac{C_{w} k_{l d} \beta^{2-\alpha_{l d}}}{\left(4 \sigma_{s} x_{m}{ }^{2}\right)^{1-\alpha_{l d}}}\left(\kappa_{1}^{\alpha_{l d}}+\kappa_{2}{ }^{\alpha_{l d}}\right)+ \\
& +\frac{C_{w n l} k_{n l} \beta^{-\alpha_{n l}}}{\left(4 \sigma_{s} x_{m}{ }^{2}\right)^{1-\alpha_{n l}}}\left(\kappa_{1}^{\alpha_{n l}}+\kappa_{2}{ }^{\alpha_{n l}}\right) .
\end{aligned}
$$

Expressions (33) and (34) can be optimized for the least costs $C_{z}{ }^{*}$ with respect to the fed zone size defined by distance $x_{m}$ (contained both in numerators and denominators of the terms); to do this, a tedious mathematical work is needed. However, it is achievable using modern technique. All quantities, except $x_{m}$ and $n=f\left(x_{m}, \Delta x\right)=\left(x_{m}-\Delta x / 2\right) / \Delta x$, are specified one way or another:

low voltage $U$ in the majority of cases is $400 \mathrm{~V}$ (given in any case);

load density $\sigma_{s}$ - by the consumers' density and their power;

$\Delta x \quad$ is the average distance between consumers;

$C_{a}$ and $C_{b}-\quad$ by the percent charges and costs of line construction;

$\gamma \quad-$ by the conductor material;

$j \quad-\quad$ by the adopted current density;

$C_{w}, C_{w n l}-$ by the price of electricity losses;

$K_{0 T f}, k_{c T} \quad-\quad$ by constant transformer facility value and specific price of transformer capacity;

$\beta \quad$ is the utilization factor of transformers;

$k_{l d} ; k_{n l} ; \alpha_{l d} ; \alpha_{n l} \quad$ - by the transformer load and no-load losses;

$\kappa_{1}, \kappa_{2} \quad$ are the power proportion ratios of the operating transformers.

In (33), the conductor cross-section can be assumed initially, and after the first optimization procedure this can be calculated by (8), (9), while the optimization by (33) is to be repeated anew.

The voltage loss can be determined using expressions from Table 2 . 


\section{MEDIUM-VOLTAGE FED ZONE}

For this stage, expressions (33), (34) are suitable, with some quantities changed. For example, expression (34) will appear as

$$
\begin{aligned}
C_{Z}^{*}= & \frac{C_{a}\left(U_{I I}\right)}{\sigma_{s} \Delta X}+\frac{2 C_{b} X_{m}}{3 \sqrt{3} j_{b} U_{I I}}\left(\frac{4 n(n+1)}{(2 n+1)^{2}}-\frac{6 n(n+1)}{(2 n+1)^{3}}\right)+ \\
& +\frac{C_{b} X_{m}}{\sqrt{3} j_{t} U_{I I}} \frac{8 n^{2}(n+1)}{(2 n+1)^{3}}+ \\
& +\frac{2 C_{w} j_{b} X_{m}}{3 \sqrt{3} \gamma_{b} U_{I I}}\left(\frac{4 n(n+1)}{(2 n+1)^{2}}-\frac{6 n}{(2 n+1)^{3}}\right)+ \\
& +\frac{8 C_{w} j_{t} X_{m}}{\sqrt{3} \gamma_{t} U_{I I}} \frac{3}{2}\left(\frac{1}{3}-\frac{n(n+1)}{(2 n+1)^{2}}\right)+ \\
& +\frac{3 n^{2}+3 n-1}{15(2 n+1)^{2}}+\frac{C_{p f} K_{0 T f}\left(U_{I I I}\right)}{4 \sigma_{s} x_{m}{ }^{2}}+\frac{C_{p f} k_{c T}\left(U_{I I I}\right)}{\beta}+ \\
& +\frac{C_{w} k_{l d} \beta^{2-\alpha_{l d}}}{\left(4 \sigma_{s} x_{m}{ }^{2}\right)^{1-\alpha_{l d}}}\left(\kappa_{1}^{\alpha_{l d}}+\kappa_{2}{ }^{\alpha_{l d}}\right)+\frac{C_{w n l} k_{n l} \beta^{-\alpha_{n l}}}{\left(4 \sigma_{s} x_{m}{ }^{2}\right)^{1-\alpha_{n l}}}\left(\kappa_{1}^{\alpha_{n l}}+\kappa_{2}{ }^{\alpha_{n l}}\right) .
\end{aligned}
$$

For higher voltage, all the quantities can change to some extent; mostly, this concerns $C_{a}$ and $K_{0 T f}$. The former value grows with voltage since the insulation class is higher, the power line towers are taller, etc. The $K_{0 T f}$ value is the greater the higher is nominal voltage $U_{I I}$ owing to stronger insulation, more costly protection and other ancillaries; $k_{c T}$ changes to a lesser degree; current density $\sigma_{s}$ changes only if there are specific consumers, otherwise $\sigma_{s}$ remains unchanged. The $\Delta X$ value changes mostly because in the higher voltage case the distance between consumers is twice the zone size of lower voltage stage (Fig. 2a):

$$
\Delta X=2 x_{m} .
$$

At a given medium voltage $U_{I I}$ the optimization procedure is the same as for the low-voltage stage; with this stage optimized, $\Delta X$ (being now the distance between transformer units) can be calculated by (36); $C_{a}\left(U_{I I}\right)$ is known; $K_{0 T f}\left(U_{I I I}\right)$ and $k_{c T}\left(U_{I I I}\right)$ can be obtained assuming the value of voltage $U_{I I I}$ of the third stage; $n=f\left(X_{m}, \Delta X\right)$. As a result of optimization, we derive size $X_{m}$ of the medium voltage fed zone (Fig. 2b).
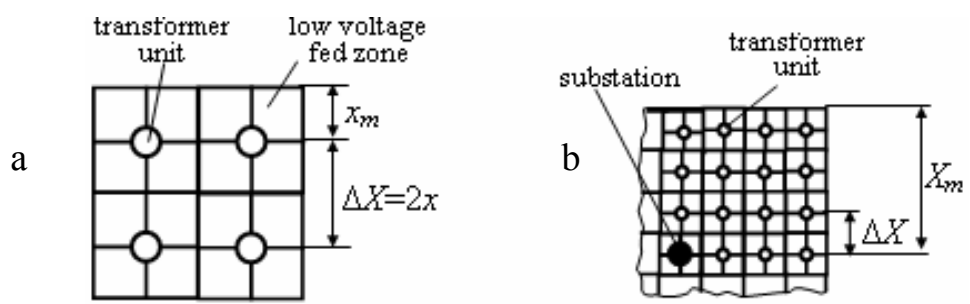

Fig. 2. Medium-voltage fed zone: $a-\Delta X$ determination; $b-$ one forth of the zone. 
Per unit cost of two stages will be the sum:

$$
C_{z+Z}{ }^{*}=C_{z}^{*}+C_{Z}^{*} \text {. }
$$

The situation changes when we can choose the value of medium voltage. Now (35) is a function of two independent variables: $U_{I I}$ and $X_{m}$. The canonical method of search for extremum is known - it is a joint solution of the equations:

$$
\frac{d C_{Z}^{*}}{d U_{I}}=0, \quad \frac{d C_{Z}^{*}}{d X_{m}}=0 .
$$

However, this method can hardly be applied due to complexity of the functions involved; also, dependences $C_{a}\left(U_{I I}\right), K_{\text {OTJ }}\left(U_{I I I}\right)$ and $k_{c T}\left(U_{I I I}\right)$ are not known. We therefore are forced to choose the relevant values from the available economic data. Nevertheless, we can evaluate the most advantageous value of $U_{I I}$ by repeated calculations for different unknown values. The question of choosing the $U_{I I I}$ value remains open. Obviously, the $U_{I I I}$ lower, the $K_{0 T f}\left(U_{I I I}\right)$ smaller, but the overall results for the $U_{I I}$ stage will be ignorable. Therefore, already in the optimization of $U_{I I}$ fed zone we should choose reasonable values of $U_{I I I}$ bearing in mind that in the stage of optimizing $U_{I I I}$ its value will be adjusted.

It should not be forgotten that in the optimization of all voltage stages the restrictions on the maximum voltage loss and current density are to be observed.

\section{THE VERSION WITH SEPARATE TRANSFORMERS}

Analyzing Eq. (33), we can see that per unit costs increase almost inversely to load density $\sigma_{s}$. At large distances between consumers the first term loses its significance as compared with the remaining terms, the voltage losses increase and the need to reduce the fourth and fifth terms by increasing voltage $U_{I}$ becomes urgent. However, the consumers would not allow increasing the voltage they use for everyday necessities. To satisfy both sides, separate transformers can be installed close to every consumer. As a criterion, the summary per unit costs of this version should be less, while the voltage losses should not exceed the allowable level. However, a separate transformer adds to per unit costs $C_{z}{ }^{*}$ of the fed zone its own per unit cost $C_{T s}{ }^{*}$, and voltage drop $U_{\Delta s e p}$ increases with that in the separate transformer $U_{\Delta T s}$. The summary quantities $C_{z s e p}{ }^{*}$ and $U_{\Delta s e p}$ are:

$$
C_{z s e p}{ }^{*}=C_{z}{ }^{*}+C_{T s}{ }^{*} ; \quad U_{\Delta s e p}{ }^{\%}=U_{\Delta}{ }^{\%}+U_{\Delta T s}{ }^{\%} .
$$

Based on Sect. 3 and Ref. [2], for a separate transformer the per unit costs $C_{T S}{ }^{*}$, transformer power $S_{s e p}$ and voltage drop $U_{\Delta T s} \%$ are:

$$
\begin{aligned}
& C_{T s}^{*}=\frac{C_{p f} K_{0 T s}}{S_{\text {sep }}}+C_{p f} k_{c T s}+\frac{C_{w} k_{l T s}}{S_{\text {sep }}^{1-\alpha_{l T u}}}+\frac{C_{w n l} k_{n T s}}{S_{\text {sep }}^{1-\alpha_{n T s}}} ; \quad S_{\text {sep }}=\sigma_{s} \Delta x \\
& U_{\Delta T s}{ }^{\%}=\frac{100 k_{r}}{U_{I}^{2} S_{\text {sep }}^{1 / \alpha_{1}}} ; \quad k_{r}=R_{T 0} \sqrt[\alpha_{1}]{S_{T 0}^{\alpha_{2}}}
\end{aligned}
$$


where $R_{T 0}$ is the resistance of a source transformer with capacity $S_{T 0}$ [2];

$\alpha_{1}, \alpha_{2}$ values are determined by selection $\left(\alpha_{1} / \alpha_{2}<1\right)$ so that $k_{r}$ found by formula coincides with the measured value for entire scale of transformer capacities.

The summary quantities (38) should not be worse than those for the version without separate transformers. Calculations have shown that, starting with load density $0.05-0.01 \mathrm{VA} / \mathrm{m}^{2}$ at large distances between customers $(200 \mathrm{~m}$ and greater, which is typical for rural regions), the application of separate transformers is justified. The per unit costs decrease while voltage losses are in permissible limits. The primary voltage of separate transformers may be in the range of $1-3 \mathrm{kV}$ (Fig. 3). Expediency of separate transformers can be tested for any set of the input data. The feasibility of using the intermediate stage should be checked by formulas of Sects. 4-6.

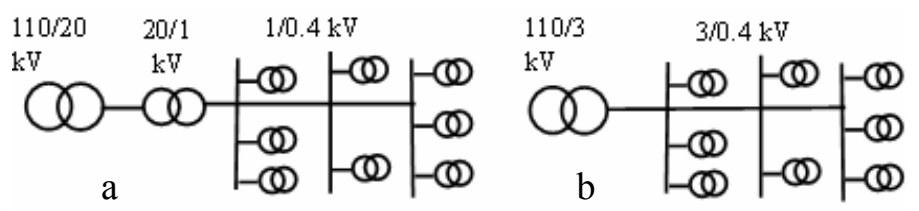

Fig. 3. Version with separate transformers: $a$ - with intermediate stage $(20 / 1 \mathrm{kV}) ; b$ - without intermediate stage.

\section{CONCLUSIONS}

1. The first term of per unit cost depends only on the load density and on the distance between consumers; in the areas with low load density it prevails over other cost terms.

2. The costs associated with power lines grow with the fed zone size, while those related to transformers decrease as the fed zone extends thus rendering a possibility to optimize its size.

3. For a given level of stage voltage it is relatively easy to optimize the size of a fed zone.

4. When the fed zone is optimized for the size and the level of stage voltage, some of the values should be assumed bearing in mind that they will be corrected when optimizing the fed zone of the next stage.

5. At low load densities and large distances between consumers it is useful to install step-down transformers directly at the consumer.

\section{REFERENCES}

1. Survilo, J. (2011). Nominal voltages of the networks. Latv. J. Phys. Tech. Sci., (2), 1326.

2. Survilo, J. (2010). The use of intermediate voltage in countryside. RTU $51^{\text {st }}$ Intern. Sci. Conf. (D:) Proceedings, 11-15 Oct., Riga (Latvia), 1-6. 
TĪKLU NOMINĀLIE SPRIEGUMI

(2. daļa)

J. Survilo

Kopsavilkums

Tiek meklēta metodika nominālo spriegumu gradācijai. Lai varētu izstrādāt izvēles metodiku, ir izveidota īpatnējo izdevumu izteiksme, kurā daudz parametru. Optizimējot šo izteiksmi, var noteikt visizdevīgākos apgādājamās zonas izmērus. Pirmie praktiskie aprēķini parādīja, ka pie maziem slodzes blīvumiem aprēķinātie fāzes vadu šḳērsgriezumi ir mazi un neatbilst iegūstamām vadu markām un tad aprēķini jāveic pie iespējamiem vadu šķērsgriezumiem. Pie noteiktiem nosacījumiem ir izdevīgi uzstādīt pazeminošos transformatorus tieši pie patērētāja.

07.10.2011. 